\title{
GW23-e2280 THE EFFECTS OF TEAM-BASED LEARNING (TBL) TEACHING USED IN CARDIOPULMONARY RESUSCITATION SKILLS TRAINING FOR THE PUBLICS
}

doi:10.1136/heartjnl-2012-302920ab.6

Ruibin Cai, Jia Xu, Yan Xiong, Hong Zhan, Zi Ye, Wei Zhan, Hong Zhan. Department of Emergency, First Affiliated Hospital of Sun Yat-Sen University

Objectives Most cardiac arrests occur outside the hospital, so it is really necessary to begin cardiopulmonary resuscitation skills training for the publics. The publics can participate in the rescue at once as the first witnesses of the cardiac arrest to improve the survival rate of the sufferer. The purpose of this research is to explore the teaching effectiveness of team-based learning teaching in cardiopulmonary resuscitation skills training for the publics.

Methods Between 15 September 2011 to 30 October 2011, 160 college students that are not medical profession participated in this research. They were trained for cardiopulmonary resuscitation skills based on 2010 CPR guidelines. Eighty students assigned into the experimental group, were trained with TBL teaching. Another 80 students assigned into the control group, were trained with traditional lecture teaching. Theory, skill-practice and cardiac arrest scene simulation, three tests were employed to evaluate the training effectiveness before the training course started and after the training course finished in two groups.

Results After training, both groups got higher scores than before training in all the tests $(p<0.05)$. College students in experimental group acted better than those in contol group in skill-practice and cardiac arrest scene simulation tests $(p<0.05)$. Better group cooperation, superior quality and higher rates of chest compression, as well as more prompt initiation of chest compression, are obtained in the experimental group $(p<0.05)$. However, there was no difference in theory test between them $(p>0.05)$. The experimental group to teaching satisfaction was also higher than those in the control group $(p<0.05)$.

Conclusions Teaching with team-based learning seems more helpful than traditional lecture teaching in training of the cardiopulmonary resuscitation skills for the publics. 